\title{
Promoting helpful attention and interpretation patterns to reduce anxiety and depression in young people: weaving scientific data with young peoples' lived experiences
}

Jennifer Y. F. Lau ${ }^{1,2^{*}}$, Rebecca Watkins-Muleba', Isabelle Lee ${ }^{1}$, Victoria Pile ${ }^{1,3}$ and Colette R. Hirsch ${ }^{1}$

\begin{abstract}
Background: Anxiety and depression are common, disabling and frequently start in youth, underscoring the need for effective, accessible early interventions. Empirical data and consultations with lived experience youth representatives suggest that maladaptive cognitive patterns contribute to and maintain anxiety and depression in daily life. Promoting adaptive cognitive patterns could therefore reflect "active ingredients" in the treatment and/or prevention of youth anxiety and depression. Here, we described and compared different therapeutic techniques that equipped young people with a more flexible capacity to use attention and/or promoted a tendency to positive/benign (over threatening/negative) interpretations of uncertain situations.
\end{abstract}

Methods: We searched electronic databases (PubMed, PsycINFO, EMBASE, and PsycARTICLES) for studies containing words relating to: intervention; youth; anxiety and/or depression and attention and/or interpretation, and selected studies which sought to reduce self-reported anxiety/depression in youth by explicitly altering attention and/or interpretation patterns. Ten young people with lived experiences of anxiety and depression and from diverse backgrounds were consulted on the relevance of these strategies in managing emotions in their daily lives and also whether there were additional strategies that could be targeted to promote adaptive thinking styles.

Results: Two sets of techniques, each targeting different levels of responding with different strengths and weaknesses were identified. Cognitive bias modification training (CBM) tasks were largely able to alter attention and interpretation biases but the effects of training on clinical symptoms was more mixed. In contrast, guided instructions that teach young people to regulate their attention or to evaluate alternative explanations of personally-salient events, reduced symptoms but there was little experimental data establishing the intervention mechanism. Lived experience representatives suggested that strategies such as deliberately recalling positive past experiences or positive aspects of oneself to counteract negative thinking.

\footnotetext{
* Correspondence: jennifer.lau@kcl.ac.uk

'Psychology Department, IOPPN, King's College London, Denmark Hill, London SE5 8AF, UK

${ }^{2}$ Youth Resilience Research Unit, Queen Mary University of London, London, UK

Full list of author information is available at the end of the article
}

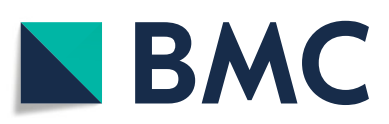

(- The Author(s). 2021 Open Access This article is licensed under a Creative Commons Attribution 4.0 International License, which permits use, sharing, adaptation, distribution and reproduction in any medium or format, as long as you give appropriate credit to the original author(s) and the source, provide a link to the Creative Commons licence, and indicate if changes were made. The images or other third party material in this article are included in the article's Creative Commons licence, unless indicated otherwise in a credit line to the material. If material is not included in the article's Creative Commons licence and your intended use is not permitted by statutory regulation or exceeds the permitted use, you will need to obtain permission directly from the copyright holder. To view a copy of this licence, visit http://creativecommons.org/licenses/by/4.0/. The Creative Commons Public Domain Dedication waiver (http://creativecommons.org/publicdomain/zero/1.0/) applies to the data made available in this article, unless otherwise stated in a credit line to the data. 
Discussion: CBM techniques target clear hypothesised mechanisms but require further co-design with young people to make them more engaging and augment their clinical effects. Guided instructions benefit from being embedded in clinical interventions, but lack empirical data to support their intervention mechanism, underscoring the need for more experimental work. Feedback from young people suggest that combining complimentary techniques within multi-pronged "toolboxes" to develop resilient thinking patterns in youth is empowering.

Keywords: Adolescence, Affective disorders, Information-processing biases, Prevention

\section{Background}

Anxiety and depression are common, disabling and frequently start in youth, underscoring the need for effective, accessible early interventions. Identifying the "active ingredients" of therapeutic change (factors, which when targeted, contribute to symptom reduction) can improve existing treatments and/or develop novel mechanismbased interventions. Equipping individuals with a more flexible capacity to use attention (enabling shifts of attention towards positive over threatening/negative information depending on circumstances) and a tendency to endorse positive/benign (over threatening/negative) interpretations of uncertain situations - could reflect active ingredients [1].

This suggestion draws on two evidence strands. Key cognitive theories propose that maladaptive attention and interpretation patterns (those focusing on or favouring threatening/negative information) maintain and increase risk for anxiety and depression [2]. Developmental cognitive neuroscience data support these theories in youth [1]. Moreover, consultations with lived experience youth representatives further corroborated the role of these cognitive factors in real-life mood fluctuations (Fig. 1), motivating and reinforcing this research focus. Second, adult treatment models of anxiety and depression show that challenging maladaptive attention and interpretation patterns and encouraging more resilient patterns is efficacious [3]. As youth involves significant growth in relevant emotion regulation abilities, adopting resilient cognitive patterns, before maladaptive ones become habitual, is fruitful [4].

Yet, outstanding questions remain on how we can most effectively target attention and interpretation patterns and whether alteration of these factors can reduce symptoms in potential treatment and prevention contexts. In this review, we describe interventions that

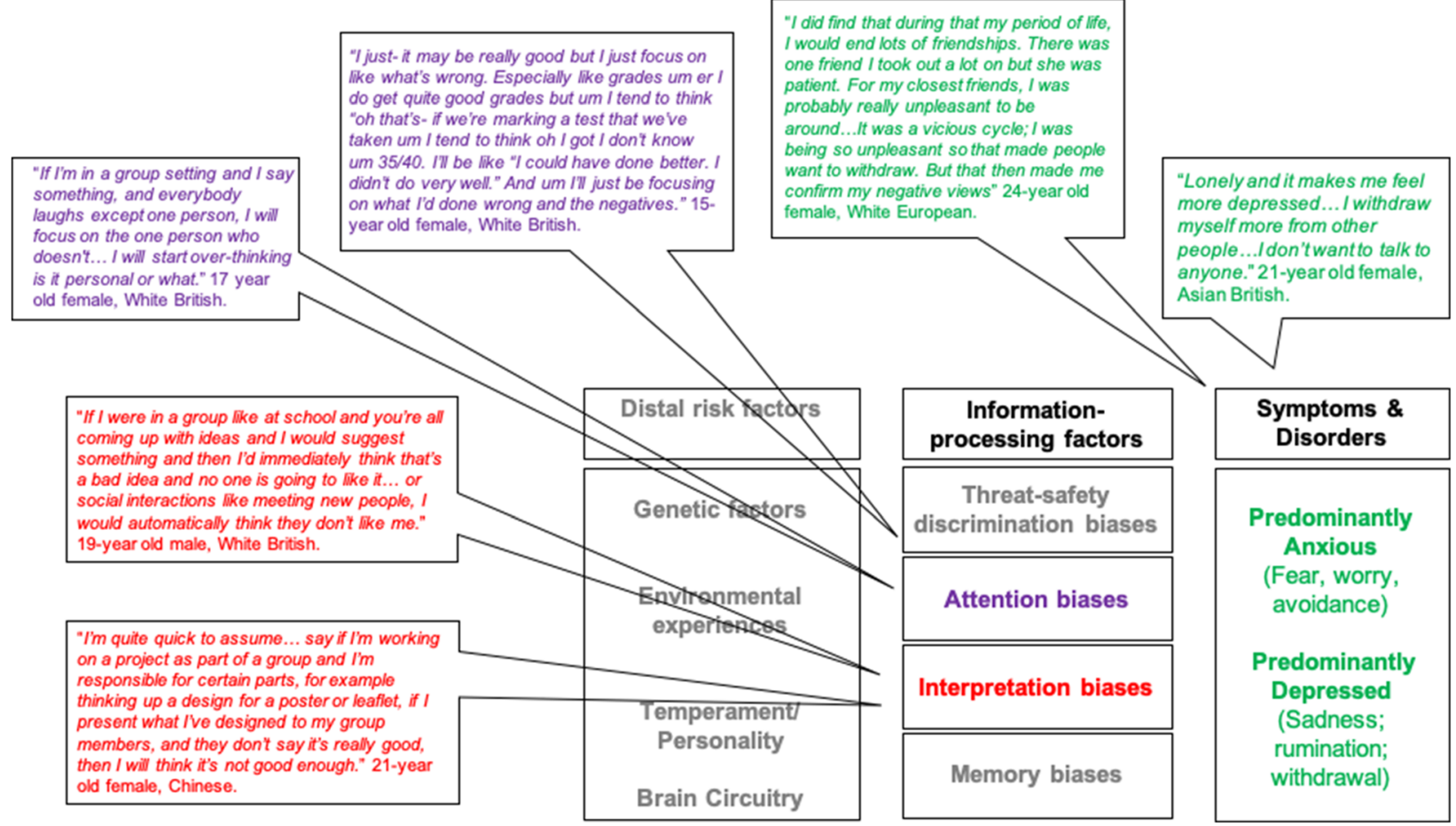

Fig. 1 An expanded information processing account of youth anxiety and depression [1] corroborated by young peoples' experiences. Information-processing biases and the role of distal factors that are beyond the scope of this report are greyed out 
explicitly target each cognitive patterns and compare their effectiveness at reducing symptoms in those with clinical anxiety/depression and those displaying high symptoms levels. Where applicable, we describe preexisting individual differences that moderate symptom reduction effects. We explore whether combined interventions targeting both processes yield greater therapeutic effects $[5,6]$. Finally, we consult lived experiences youth representatives over their experiences of using helpful thinking patterns in daily life, their effectiveness and how these techniques could be further enhanced.

\section{Method}

\section{Systematic review}

Our review was pre-registered at PROSPERO (CRD42020196651). Following published guidelines for the Preferred Reporting Items for Systematic Reviews and Meta-Analyses (PRISMA) [7], in June/July 2020, we searched electronic databases (PubMed, PsycINFO, EMBASE, and PsycARTICLES) for studies containing words relating to: intervention; youth; anxiety and/or depression and attention and/or interpretation. Titles, abstracts and where required, full texts were screened to determine inclusion in the review by two psychology students (IL, RWM), with a smaller subset (10\%) reviewed by the lead author JL. We applied the following inclusion criteria: 1) peer-reviewed original research published in English-language journals; 2) investigated human participants with a mean age between 14.0 and 24.9 years; 3 ) reported on interventions or manipulations that sought to reduce self-reported anxiety/depression by explicitly altering attention and/or interpretation patterns.

For each paper, we recorded: publication details, participant numbers and demographics in the intervention and comparison group, primary presenting problem (anxiety/depression/both) and how this was assessed (diagnostic interview/questionnaire), and the intervention and comparison condition characteristics (dosage/ delivery mode). Effect sizes of any pre-to-post anxiety/ depression symptom change in the intervention group and the between-group post-intervention symptom difference between intervention and comparison group participants were included/calculated. Heterogeneity across study samples, designs, and intervention characteristics warranted a narrative synthesis rather than meta-analyses.

\section{Consultations with lived experience representatives}

Ten young people (aged 15 to 24 years; 7 females; 6 White British) with varying severity of past anxiety and depression (and treatments) were invited to provide consultation about the relevance of managing unhelpful thinking styles in daily life. Efforts were taken to encourage diversity in age range, gender and ethnicity.

Young people were invited through online advertisements on dedicated research websites. A topic guide was developed for consultation sessions, which occurred as one-to-one meetings with the researchers (JL, VP). This topic guide asked young people to think about situations that provoked feelings of anxiety and depression, the thinking patterns that amplified negative emotions, and strategies they took to challenge unhelpful thinking patterns. Young peoples' perspectives on how thinking patterns could amplify negative emotions are presented in Fig. 1 and served as a rationale for our systematic search. However, their thoughts on strategies used to challenge unhelpful thinking patterns were used to complement the findings of our systematic search.

\section{Results}

Study selection is summarised in Fig. 2. Of the final 80 papers, 22 studies modified attention patterns; 52 , interpretational patterns; and 6, targeted both. All studies were used to address the range and effectiveness of interventions targetting attention and interpretational patterns. To address symptom reduction effects, only studies involving "clinical" participants (those reporting diagnoses of anxiety/depression) and "high-symptom" participants (those selected on the basis of symptoms above a clinical cut-off or in relation to other participants) were used. For these studies, we only included those that involved at least two intervention sessions as many early studies (even involving clinical/high-symptom participants) were designed to test for the plasticity of cognitive factors and causal links with symptom change in the short-term (within-session).

\section{Interventions promoting helpful attention patterns Attention Bias modification interventions}

Of 22 attention studies [8-28], nineteen (86\%) used Attention Bias Modification (ABM) training to shift (and reinforce) maladaptive attention patterns away from threatening/negative to neutral or positive stimuli across multiple training trials. Common training tasks are the visual dot-probe and visual search training tasks [29] (Fig. 3). The visual dot-probe (and its' variants) train the orienting of attention away from threatening/negative cues towards neutral/positive cues, by presenting response-probes more frequently behind neutral/positive stimuli. The visual search task promotes goal-directed attention, by instructing participants to identify positive stimuli from an array of competing threatening/negative stimuli. Across studies, ABM training was delivered between 1 and 13 sessions. Most studies employed a similar computerised task not designed to modify attention as an active comparison condition. For the visual dot- 


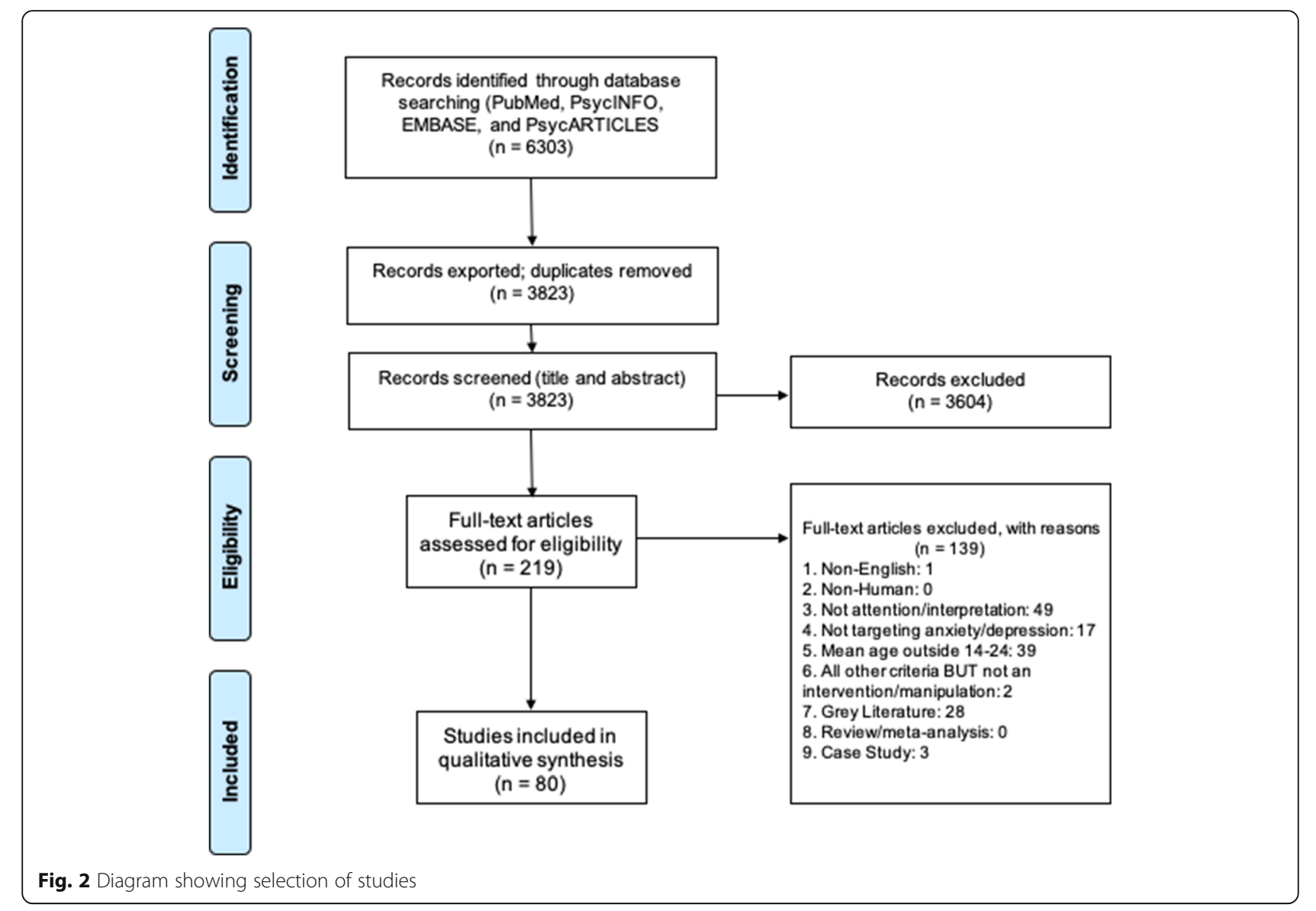

probe, this control condition involved responding to a probe that appeared with equal frequency behind a threatening/negative versus neutral/positive stimuli. In the visual search control task, participants searched for a 5-petalled flower from 7-petalled flowers. Most studies delivered $\mathrm{ABM}$ via computers, but smartphone methods were also trialled [30].

Ten ABM studies reported significantly greater reduction of attention biases for threatening/negative information in the intervention than the comparison condition $[8,12,17,18,20-24,26]$. Three other studies reported expected changes on attention bias but only when particular variants/versions of $\mathrm{ABM}$ were used (visual search [25], spatial cueing [23]); or under specific combinations of stimuli by exposure conditions [14].

\section{Attention flexibility interventions}

Two studies $(27,28,9 \%)$ aimed to improve general Attention Flexibility (AF, Fig. 3) using the Attention Training Technique (ATT) [31] and/or a Mindfulness-based intervention. ATT is designed to strengthen the ability to flexibly use and control attention through explicit instruction [31]. Across training phases, participants engage in selective attention, attention switching and dividing their attention between neutral (e.g. sounds) stimuli in the environment. Mindfulness-based interventions can also target general attention regulation [32]. Exercises may benefit concentration (the sustained aspect of attention), effortful attention-inhibition of distracting information, goal-directed attention control, and flexible switching of attention. While the focus is usually on neutral stimuli or internal sensations, these exercises also involve 'sitting with' more unpleasant sensations (e.g. pain) in non-judgmental way.

Only one of the two studies targeting AF collected measures to assess changes in attention patterns [28]. Participants receiving ATT or mindfulness showed similarly large (significant) pre-to-post increases in questionnaire measures of attention flexibility.

\section{Symptom reduction effects of promoting helpful attention patterns $A B M$ interventions}

Three multisession visual dot-probe $\mathrm{ABM}$ studies were conducted in clinical samples (Table 1). One involving young people with Social Anxiety Disorder [10] found small within-(intervention)-group reductions in symptoms from pre-to-post-intervention, but only $\mathrm{ABM}$ 


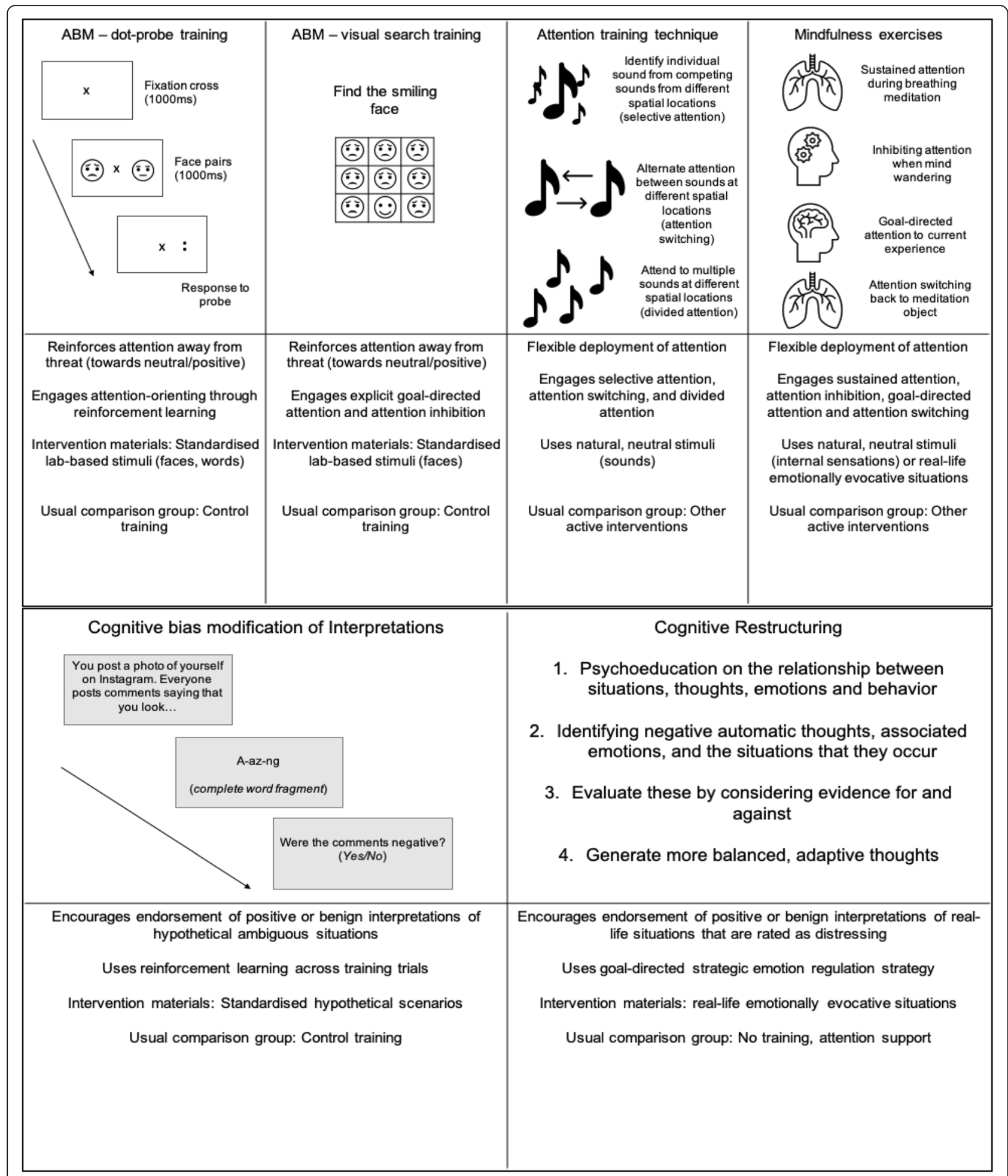

Fig. 3 Summary of interventions promoting helpful attention (top panel) and interpretation patterns (bottom panel)

participants showed continued decreases to a 3-month follow-up. The absence of significant reductions in attention biases suggests that symptom changes were not driven by measurable changes in attentional patterns.
Interestingly, this study noted that within adolescents allocated to receive ABM, those with higher trait attention control (reported by parents) showed significantly lower social anxiety symptoms at post-intervention. ABM was 
Table 1 Promoting helpful attention patterns in participants with clinical symptoms (in bold) and high symptom scores (not bold). Where studies do not give separate demographic information for each group, these are combined in a single cell. Where effect sizes were not reported or could not be calculated, these are labelled as Not Reported (NR). Green highlighted rows reflect studies showing large within group symptom reduction and at least medium sized between group effects. Orange highlighted rows reflect studies showing near large within group symptom reduction and small sized or non-reported between group effects

\begin{tabular}{|c|c|c|c|c|c|c|c|}
\hline Authors (year) & $\begin{array}{l}\text { Presenting } \\
\text { problem of } \\
\text { sample }\end{array}$ & \begin{tabular}{|l|} 
Intervention \\
group number of \\
participants; \\
gender \\
proportion, \\
ethnicity, age \\
range
\end{tabular} & $\begin{array}{l}\text { Control group } \\
\text { number of } \\
\text { participants; } \\
\text { gender } \\
\text { proportion, } \\
\text { ethnicity, age } \\
\text { range }\end{array}$ & $\begin{array}{l}\text { Number of } \\
\text { sessions }\end{array}$ & $\begin{array}{l}\text { Nature of control } \\
\text { group }\end{array}$ & $\begin{array}{l}\text { Within group } \\
\text { effect size of } \\
\text { pre-to-post } \\
\text { primary } \\
\text { symptom } \\
\text { measure } \\
\text { change }\end{array}$ & $\begin{array}{l}\text { Between-group } \\
\text { effect size of } \\
\text { post-intervention } \\
\text { primary } \\
\text { symptom } \\
\text { measure }\end{array}$ \\
\hline \multicolumn{8}{|c|}{$\frac{1}{\text { Attention Bias Modification Training }}$} \\
\hline Ollendick et & Social Anxiety & $\mathrm{N}=29$ & $\mathrm{~N}=29$ & 10 & Control training & 0.25 & 0.09 \\
\hline al., 2019 & Disorder & $\begin{array}{l}31 \% \text { male; } \\
79 \% \text { Caucasian; } \\
\text { Mean } \\
\text { age }=14.34\end{array}$ & $\begin{array}{l}28 \% \text { male; } \\
90 \% \text { Caucasian; } \\
\text { Mean age }=14.24\end{array}$ & & & & \\
\hline $\begin{array}{l}\text { Rieman et al., } \\
2013\end{array}$ & $\begin{array}{l}\text { Anxiety } \\
\text { disorders }\end{array}$ & $\begin{array}{l}\mathrm{N}=21 ; \\
48 \% \text { male } \\
100 \% \\
\text { Caucasian; } \\
\text { Mean } \\
\text { age }=15.43\end{array}$ & $\begin{array}{l}\mathrm{N}=21 ; \\
48 \% \text { male; } \\
90 \% \text { Caucasian } \\
\text { Mean age }=15.71\end{array}$ & NR but $>1$ & Control training & 1.43 & 0.64 \\
\hline \multirow[t]{2}{*}{$\begin{array}{l}\text { Yang et al., } \\
2016\end{array}$} & \begin{tabular}{|l} 
Major \\
Depressive \\
Disorder
\end{tabular} & $\begin{array}{l}\mathrm{N}=23 ; \\
48 \% \text { male; } \\
\text { Ethnicity: NR }\end{array}$ & $\begin{array}{l}\mathrm{N}=22 ; \\
41 \% \text { male; } \\
\text { Ethnicity: NR }\end{array}$ & 12 & Control training & 2.77 & 0.63 \\
\hline & & $\begin{array}{l}\text { Mean } \\
\text { age }=15.09\end{array}$ & Mean age $=14.82$ & & & & \\
\hline $\begin{array}{l}\text { Maoz et al., } \\
\text { (2013) }\end{array}$ & $\begin{array}{l}\text { Social Anxiety } \\
\text { symptoms }\end{array}$ & $\begin{array}{l}\mathrm{N}=24 \\
\text { Males: } 17 \% \\
\text { Ethnicity: N/R } \\
\text { Mean Age= } \\
22.96\end{array}$ & $\begin{array}{l}\mathrm{N}=26 \\
\text { Males: } 23 \% \\
\text { Ethnicity: } \mathrm{N} / \mathrm{R} \\
\text { Mean Age= } \\
22.42\end{array}$ & 4 & Control training & 0.07 & 0.05 \\
\hline $\begin{array}{l}\text { Yao et al., } \\
\text { (2015) }\end{array}$ & $\begin{array}{l}\text { Social Anxiety } \\
\text { symptoms }\end{array}$ & $\begin{array}{l}\mathrm{N}=23 \\
\text { Males: } 43.5 \% \\
\text { Ethnicity: } 100 \% \\
\text { Chinese } \\
\text { Mean Age= } \\
20.87\end{array}$ & $\begin{array}{l}\mathrm{N}=23 \\
\text { Males: } 26.1 \% \\
\text { Ethnicity: } 100 \% \\
\text { Chinese } \\
\text { Mean Age= } \\
20.09\end{array}$ & 4 & Control training & 0.03 & 0.04 \\
\hline \multirow[t]{2}{*}{$\begin{array}{l}\text { Pan et al., } \\
2019\end{array}$} & $\begin{array}{l}\text { Social Anxiety } \\
\text { symptoms }\end{array}$ & $\begin{array}{l}\mathrm{N}=20 \\
10 \% \text { male; }\end{array}$ & $\begin{array}{l}\mathrm{N}=20 \\
15 \% \text { male; }\end{array}$ & 8 & Control training & 0.49 & 0.31 \\
\hline & & $\begin{array}{l}\text { 100\% Chinese: } \\
\text { Mean } \\
\text { age }=20.05\end{array}$ & $\begin{array}{l}100 \% \text { Chinese; } \\
\text { Mean age }=19.94\end{array}$ & & & & \\
\hline
\end{tabular}


Table 1 (Continued)

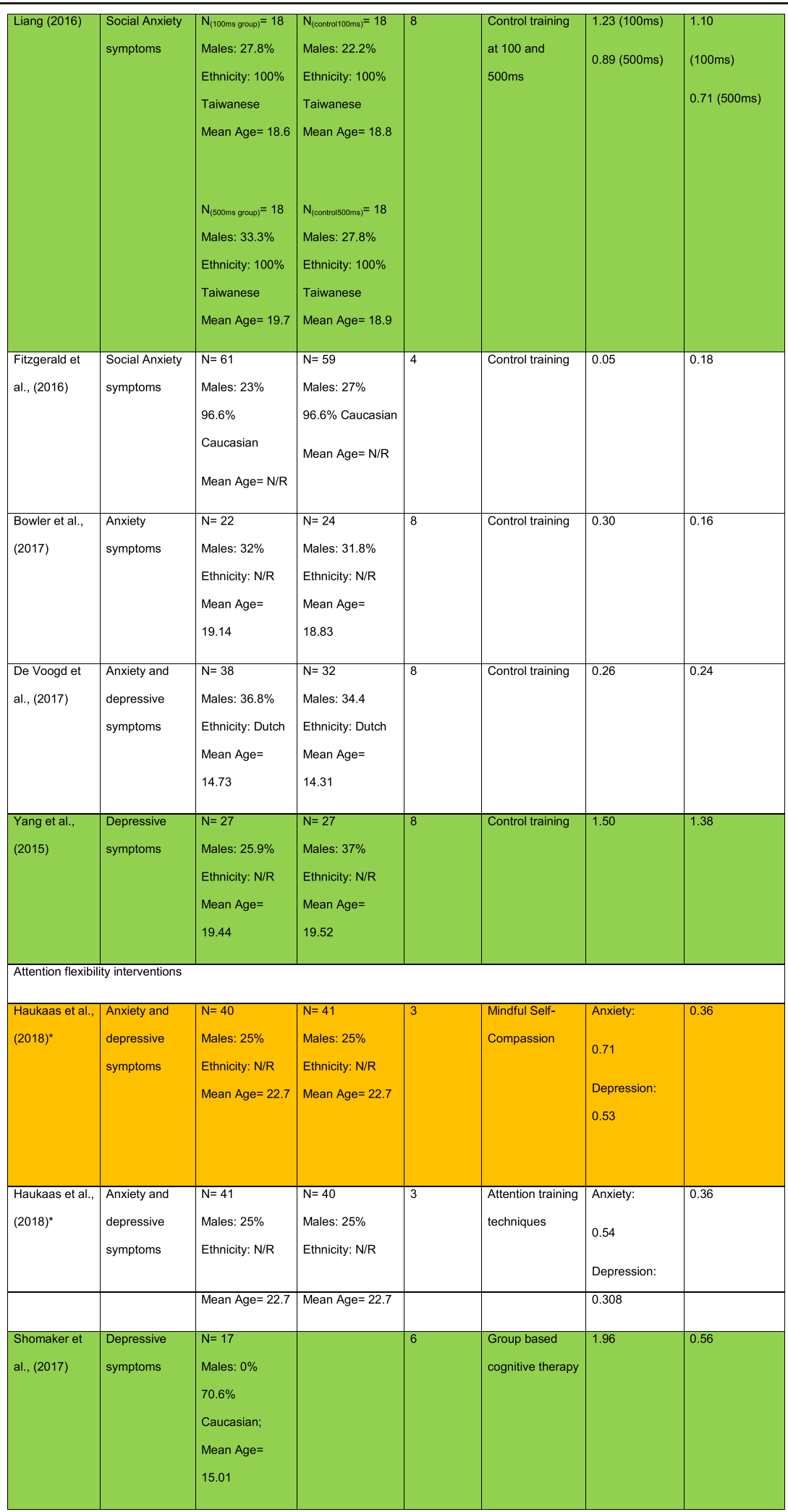

*Study compared attention training technique against a mindfulness-based intervention; each of these groups is entered as a different row to show within-group changes 
also assessed as a way of augmenting the effects of Cognitive Behavioural Therapy (CBT) in young people with complex forms of anxiety [9]. Those who received ABM showed large within-group symptom reduction with large differences to the control-training-plus-CBT group at postintervention. The authors did not report scores on attention bias measures making it difficult to attribute large symptom reduction effects to changes in attention patterns. Working with young people with depression [8], one study reported large within-group symptom reduction in the intervention group and a large group difference with control participants post-intervention (effects that persisted to a 12-month follow-up). Importantly, there were greater reductions in attention bias scores among those receiving $\mathrm{ABM}$ than the control condition, suggesting that symptom change could be due to attention change.

Eleven ABM studies were conducted in high-symptom participants (Table 1). Seven involved young people with social or general anxiety symptoms [11-14, 16-18]. With one exception [14], symptom reduction effects from preto-post intervention in the $\mathrm{ABM}$ training condition were small across studies (Cohen's $d=0.03-0.49$ ). Two [20, 21] (of the 4) studies targeting depression reported moderateto-large size reduction in symptoms in the ABM condition (at post-intervention and follow-up time-points) that were linked with significant changes in attention bias. However only one of these reported a significant difference to their comparison condition at post-training and follow-up assessments [20]. One study reported no differential reduction of depression symptoms in the ABM compared to control group [15], and unexpectedly, one study [19] found greater symptom decreases in control participants.

\section{AF interventions}

In a comparison of ATT with mindfulness in young people with high anxiety/depression symptoms [28], both groups showed medium-sized improvements, which were maintained at 6 months. Improvement in questionnaire reports of attention flexibility, significantly predicted treatment response. The second study [27] compared a mindfulness-based intervention with CBT in young people with depressive symptoms at-risk for Type 2 diabetes. Greater symptom reduction occurred in the mindfulness group than the CBT group at post-treatment (Cohen's $\mathrm{d}=0.56)$ and at 6 months follow-up (Cohen's $\mathrm{d}=0.69$ ). No attention process measures were collected.

\section{Interventions promoting helpful interpretation patterns} Cognitive Bias modification of interpretations interventions Fifty-two studies [17, 18, 27, 30, 33-81] modified interpretations. Twenty-three (44\%) included Cognitive Bias Modification of Interpretations (CBM-I) training. Similar to $\mathrm{ABM}$, this uses repeated reinforcement learning to encourage the endorsement of positive (or benign) interpretations of ambiguous information, over-riding the tendency to infer threatening/negative explanations. A common training task presents individuals with incomplete written ambiguous situations; completion of a word fragment resolves ambiguity in a positive/benign direction (Fig. 2). Most studies present incomplete emotionally-neutral situations as an active comparison condition. The number of training sessions across clinical, high-symptom and unselected studies ranged from single-sessions to 15 .

Of 23 studies, 18 (78\%) reported clear training effects in the CBM-I intervention condition, that is, either decreased negative interpretations or increased benign/positive interpretations from pre-to-post intervention or relative to the comparison condition at post-intervention. However, most studies used a measure of interpretational style that was structurally similar to the training task and could reflect demand effects. Where studies assessed transfer effects using a different measure of interpretation style, training effects in the CBM-I condition were mixed [33, 37, 54].

\section{Cognitive restructuring interventions}

Thirty-one studies (59\%) used Cognitive Restructuring (CR). This uses explicit instruction (Fig. 2) to encourage individuals to generate alternative explanations for situations and to consider evidence for and against each explanation. $\mathrm{CR}$ is a routine component in many CBT protocols for youth anxiety/depression, but can be used as a standalone intervention. Varying between 8 and 16 sessions, CR can be administered in individual or group sessions, face-to-face or remotely. One study [74] used Cognitive Reappraisal training, a version of CR focused on teaching participants to re-interpret distressing situations, sometimes through a third-party perspective ("psychological distancing").

Seven of the 31 studies collected measures to inform changes in cognitive patterns, but none directly measured interpretation style. Yet, all 7 showed expected changes in the intervention compared to the comparison condition, which could reflect the products of increased positive/benign interpretation of daily situations. One reported changes in adolescents' estimation of certain anxietyprovoking events [40]. Another reported reductions in irrational beliefs [61]. Decreases in automatic negative thoughts and increases in automatic positive thoughts [52] as well as decreases in self-negative statements [46, 62], and increases in positive cognitions around hypothetical stressful situations [79] were reported.

\section{Symptom reduction effects of promoting helpful interpretation patterns $C B M-I$ interventions}

Two studies (Table 2) delivered multisession CBM-I training to young people with clinical depression [33, 34], reporting small-to-moderate symptom reduction changes 
Table 2 Promoting helpful interpretation patterns in participants with clinical symptoms (in bold) and high symptom scores (not bold). Where studies do not give separate demographic information for each group, these are combined in a single cell. Where effect sizes were not reported or could not be calculated, these are labelled as Not Reported (NR). Green highlighted rows reflect studies showing large within group symptom reduction and at least medium sized between group effects. Orange highlighted rows reflect studies showing near large within group symptom reduction and small sized or non-reported between group effects

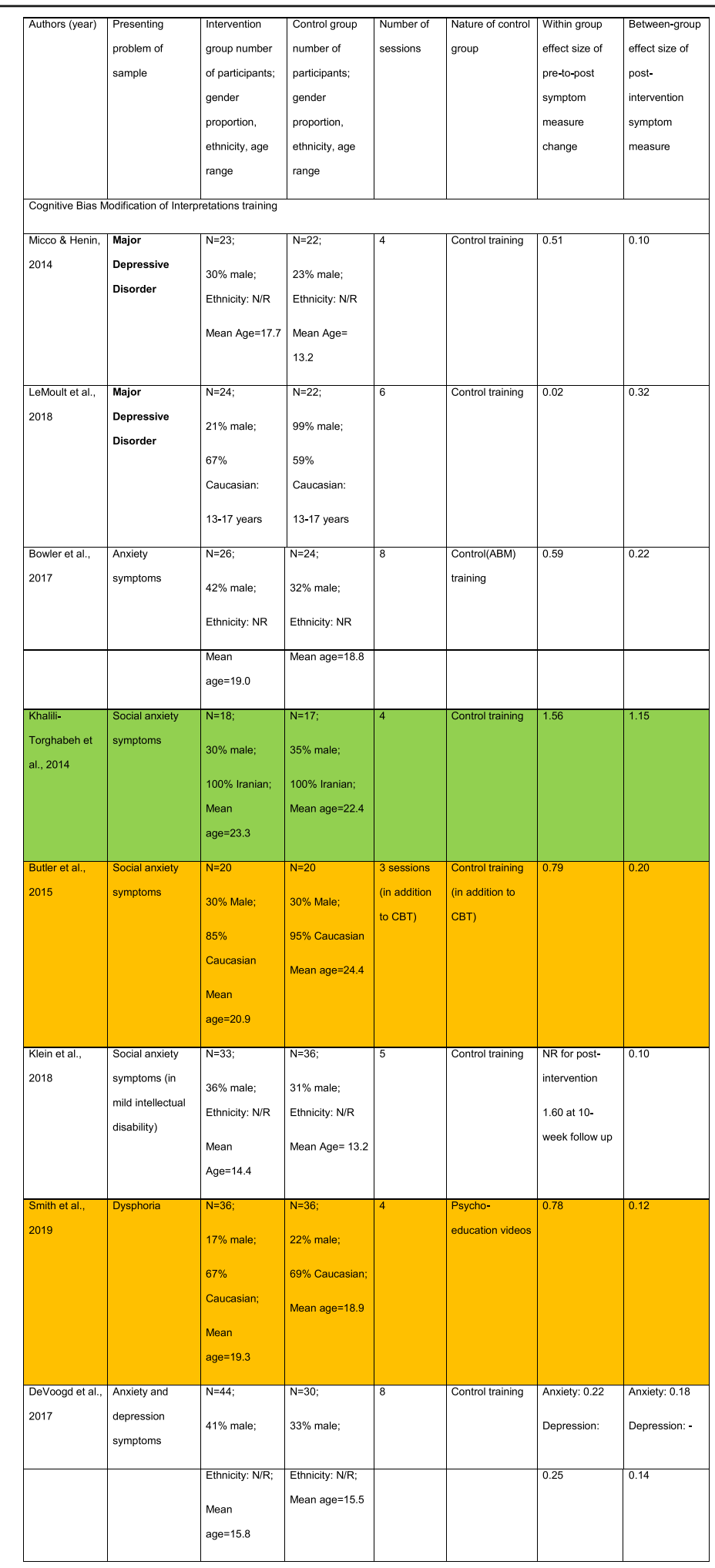


Table 2 (Continued)

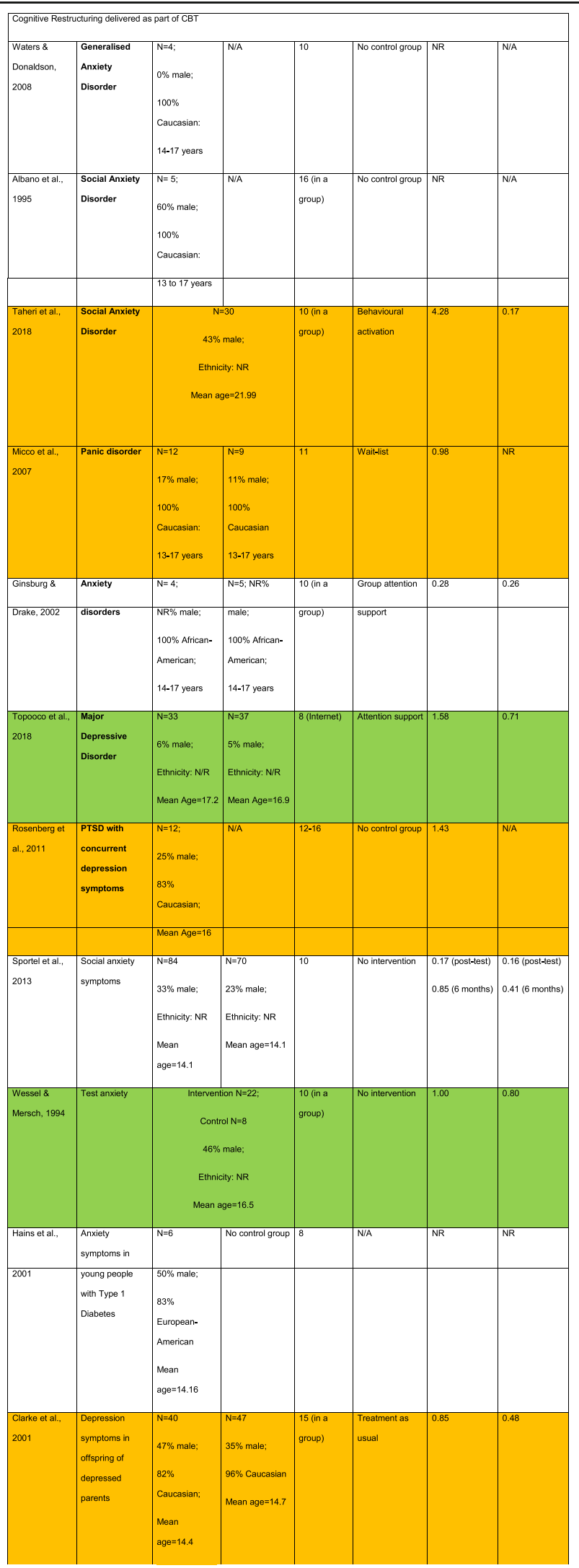


Table 2 (Continued)

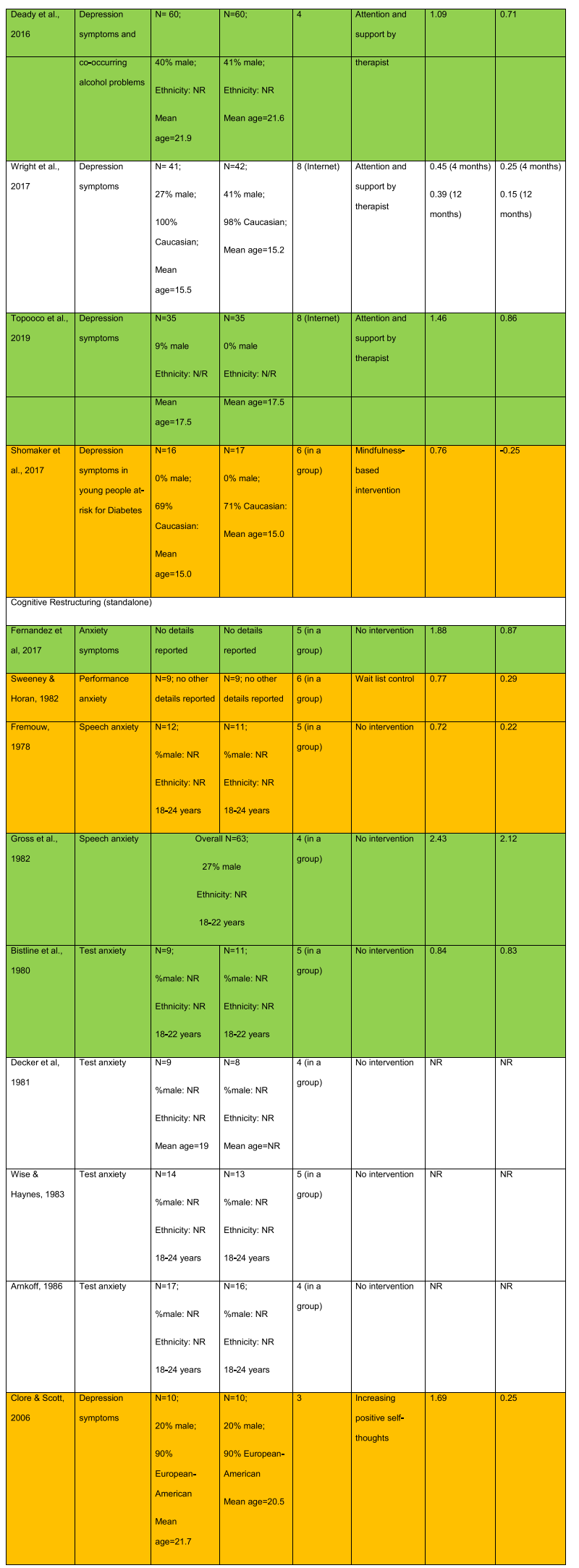


in the intervention group (Cohen's $d=0.02 / 0.51$ ). There were also small differences post-intervention with the control condition (Cohen's $d=0.10 / 0.32$ ). No studies delivered multi-session CBM-I training to young people meeting diagnostic criteria for an anxiety disorder.

Six studies applied CBM-I to young people with high symptom scores (Table 2). Only one reported large symptom improvement in the CBM-I group, and equally large differences compared to a control condition, with expected changes in interpretational style [35]. Three studies of general anxiety [18], social anxiety [36] and dysphoria [38] showed medium-sized symptom reduction in the CBM-I group (Cohen's $d=0.59-0.79$ ) and small between-group differences with various comparison conditions post-intervention (Cohen's $d=0.12$ 0.22). Finally, two studies targeting social anxiety [37] or anxiety/depression [17] reported small within-group symptom reduction and small post-intervention differences with the comparison condition. However, for one, CBM-I training effects on symptoms were more apparent at 10-weeks (Cohen's $d=1.60$ ) [37], with expected changes in positive interpretation.

\section{$C R$ interventions}

Seven studies assessed CR techniques within CBT in young people with clinical anxiety and/or depression, as the primary condition or co-morbid with PTSD (Table $2)$. Three were case series $[39,43,81]$, where no data on symptom measures was reported, or were based on fewer than 5 participants. Two studies targeting anxiety disorders (social anxiety [41], panic [40]) showed large reductions in symptoms from pre-to-post intervention in the CR condition. Micco and colleagues [40] recorded session-to-session change on anxiety, and noted a therapeutic gain following the first session of CR. However, in both studies, there was either no data reported from the (wait-list) control group, or the control group was another active intervention (behavioural activation [41]), in which case the between-group difference at postintervention on anxiety symptoms was small. Using CR to target depression, two studies revealed large withingroup symptom reduction effects [42,44], but only one employed a comparison condition (comprising monitoring and non-specific counselling), and reported a moderate sized post-intervention difference in depressive symptoms [42].

Nine studies employed CR (within CBT) in young people with high symptom levels. Two assessed CR effects on anxiety symptoms with one finding weak [45], and the other strong [46], within-group reduction effects. The study reporting the stronger within-group changes noted a reduction in negative self-statements [46]. A case series aiming to reduce anxiety symptoms in young people with a chronic health condition [47] did not report whole-sample mean changes but all 6 participants improved across treatment. For the 6 studies assessing depression symptoms (with one targeting depression in a sample at-risk for a chronic health condition [27]), 4 reported large within-intervention-group pre-to-post symptom reduction effects and medium-tolarge between-group differences with a comparison condition (treatment as usual or attention support) at postintervention [42, 48-50]. The two other studies reported weak or medium-sized symptom reduction effects [27, 51]; the one reporting smaller-sized changes assessed this at 4 months [51] so improvements may have become weak with time.

Nine studies examined standalone CR interventions in young people with high general anxiety/depression symptoms or with specific test, speech or performance anxiety (Table 2). Three noted significant reductions in anxiety measures from pre-to-post-intervention in the CR condition but did not report enough data to calculate effect sizes [58, 60, 61]. Where effect sizes were reported, within-group symptom reduction were medium to large (Cohen's $d=0.72-2.43$ ). Two studies that reported large effect sizes also found expected changes in automatic negative thoughts and negative/positive self-statements in the CR group [52, 62]. Comparisons with waitlist/nointervention conditions across studies showed weak to large between-group differences at post-intervention. One study noted that individuals with lower purposeful engagement (the reduced tendency to attend/engage with unpleasant thoughts) benefited more from CR techniques than those receiving the comparison condition [44].

\section{Amplifying the effects of attention and interpretation interventions \\ Combined interventions}

Six studies jointly targeted attention and interpretation patterns in reducing anxiety/depression. Four involved clinical participants. The first [82] delivered a web-based intervention combining $\mathrm{CR}$ and ATT in reducing social anxiety. Effect sizes (Cohen's d) for the intervention group were $0.72-0.82$ on symptoms from baseline to a 4-month follow-up, and were significantly greater than changes reported in the wait-list control group. Piet and colleagues [83] combined mindfulness-based cognitive therapy with CBT in socially-anxious young adults, thus targeting $\mathrm{AF}$ and $\mathrm{CR}$. Combining interventions yielded greater within-group symptom reduction but this increase was marginal compared to receiving one treatment (Cohen's $d=0.20-0.33$ ). O'Toole and colleagues [84] applied Emotion Regulation Therapy to young people with generalised anxiety disorder, cultivating AF (shifting and sustaining attention on a difficult experience) and CR abilities. Within-intervention-group reductions emerged on anxiety symptoms (Cohen's $d=1.2-$ 
1.4), preceded and mediated by changes in cognitive reappraisal and decentring, a cognitive skill, inversely correlated with negative self-referential processing. Finally, a one-day group-based CBT package was delivered to 24 young people with clinical anxiety/depression [85]. Of 8 workshop topics, one corresponded to $\mathrm{CR}$ and one to AF (within mindfulness). The study aimed to gather qualitative feedback from young people. One theme that emerged was that the wide selection of techniques enabled young people to learn a suitable technique.

Two case series $[86,87]$ piloted a combined ABM and CBM-I intervention to reduce high anxiety/depression symptoms. Neither included a comparison condition and neither was powered to detect significant withingroup changes. The first showed reduction of social anxiety symptoms in around $80 \%$ of participants. Using a similar intervention but adapted/translated for young people with a history of victimisation in the UK and Nepal, social anxiety scores reduced only in UK participants (Cohen's $d=0.81$ ). Qualitative feedback across both studies suggested poor engagement with $A B M$ than CBM-I training.

\section{Consultations with lived experience representatives}

Young peoples' perspectives on how thinking patterns could amplify negative emotions were used to support our research focus (Fig. 1). However, several more messages emerged from discussing the use of helpful cognitive patterns in daily life, barriers and how these could be enhanced.

First, participants spoke about adopting alternative perspectives, such as those used in CR or CBM-I, as being useful in daily life. One young person also noted that this was especially using a third-person perspective.

"Yeah so you don't think all the focus is on you ... you could say something from a positive angle like they could be talking about somebody else not talking about you." 21-year old female, Asian British.

"The therapy helped me by not having the negative thoughts. making me aware of it ... talking about this, is it really the person or is it me, or getting another perspective ... rather than cutting the relationship has helped" 24-year old female, White European.

"Challenging those thoughts do [es] help, especially trying to find pieces of evidence to go against what I think ... It is also helpful to get someone else to suggest alternative perspectives" 21-year old female, Chinese.

However, some noted obstacles in current interventions, underscoring a need to help young people discover methods for learning and implementing helpful cognitive patterns:

"In counselling, they try to think about what is and isn't irrational. And try to think about all the positive explanations before you jump to negatives ... if I can recognise it is happening this can be easier, but very often I don't so it doesn't help massively". 17year old female, White British.

To improve their effectiveness, one young person suggested using both attention and interpretation patterns to manage negative emotions:

"I think someone once told me that a thought only lasts for 8 seconds unless you chose to prolong it yourself. For me, I find it easier to engage it a little more and find out where it has come from, why do I think this, where has it come from, and then challenge the idea in my head" 19-year old male, White British.

Young people also described that the deliberate recall of positive past experiences or positive aspects of oneself could help to counteract negative thinking:

\begin{abstract}
"May be if I try to point out the things that are good and maybe compare it to another piece of artwork I've done before. Or maybe think "well you've struggled on this but look now, you've managed to do this better than you've done before"' 15-year old female, White British.

"I write down things I appreciate about myself ... Say if I cooked dinner for my friends, they didn't give that much positive feedback, I might automatically assume my cooking wasn't that good or I had not cooked enough food. But then if I wrote down that I actually cooked for them, I might feel better." 21year old female, Chinese.
\end{abstract}

\section{Discussion}

Helpful attention and interpretation patterns can potentially help young people better navigate daily-life emotional situations. As youth is a time in which maladaptive cognitive patterns and their links with anxiety/depression consolidate $[88,89]$, implementing more resilient cognitive responses can divert away from negative trajectories. Our search of the scientific literature revealed a myriad of techniques: these could be differentiated by whether they target attention or interpretation (or both) but they could also be divided into bias modification training techniques (ABM, CBM-I) versus instructed, strategic, goal-directed techniques. Our findings show interventive potential for 
each category whether targeting attention or interpretation or whether directed at clinical or sub-clinical symptoms. Here, we summarise these findings along with messages from consultations with young people and suggest necessary research to realise these areas of intervention potential, within this quickly expanding field.

\section{$A B M$ and $C B M-I$ techniques}

Emerging from cognitive science research, bias modification training studies were designed to target a specific mechanism and its causal link with symptoms. Thus, studies usually include a well-matched comparison condition and pre to post-measures of the intended mechanism, enabling one to draw inferences over the cognitive origins of symptom reduction. Given these dedicated efforts to target the mechanism, $A B M$ and CBM-I studies broadly showed changes in bias (although questions remain over the poor reliability of reaction time based measures of attention bias and transfer effects of training to other interpretation bias measures). Less observed were consistently medium or large reductions in symptoms either in clinical or high-symptom groups following ABM/CBM-I. However, these training paradigms were not designed to engage clients in multisession interventions. Training is presented on a computer using lab-developed stimuli (faces, words) with no psychoeducation rationale for the intervention. Training dosage is somewhat arbitrarily determined and the infrequent inclusion of follow-up assessments makes it difficult to assess whether symptom changes occur only after consolidation of training.

To address these issues, adult and pre-adolescent ABM studies have begun to base new training paradigms on visual search protocols more, as these may more efficiently change maladaptive attention patterns by enhancing the voluntary capacity to select positive/benign stimuli [29]. Implementations have shown consistent symptom reduction $[90,91]$. In adults, a version of the visual search training paired with reinforcement incentives (e.g. music that plays when looking at smiling faces over negative faces), has yielded strong training and symptom reduction effects in adults with social anxiety [92] and major depression [93]. A further advantage is the use of eye-tracking to monitor and measure gaze patterns, yielding more reliable and valid assessments of attention patterns across time. While this promising "gaze-contingent music reward therapy" has been trialled in pre-adolescents [94], it has not been assessed in young adults. For CBM-I, symptom reduction effects could be augmented by tailoring materials to the day-today lives of the targeted population to increase engagement and generalisation to real life. Adult research also suggests the use of prolonged imagery to self-generate outcomes during training to scaffold training effects
[95]. Harnessing virtual reality within training could also be fruitful in engaging young people [96]. If we are to discover the clinical potential of these mechanism-based training interventions, it will be crucial to address issues around user-engagement, co-designing these with young people.

\section{Attention flexibility and cognitive restructuring interventions}

Unlike bias modification training, AF and CR techniques have developed over decades, as part of complex cognitive-behavioural interventions. These use naturalistic stimuli or real-life scenarios to embellish learning, are guided by clinical insights over dosage, and evaluated as part of larger trials with lfollow-up assessments, increasing the opportunity to demonstrate clinical effects. Here, both $\mathrm{AF}$ and $\mathrm{CR}$ interventions were associated with consistent medium/large symptom reduction effects in across clinical and high-symptom participants. However, as these broader complex intervention programmes target many other treatment components, use either no or poorly matched comparison groups and often do not include direct measures of attention and interpretation patterns, it is difficult to attribute symptom reduction to changes in cognitive patterns. Isolating "active ingredients" of symptom reduction is important for developing brief, accessible, scalable and transportable interventions. Thus, the next wave of research should focus on delineating the mechanisms underlying these interventions. Grounding these within rigorous experimental studies that use active comparison conditions to control for other non-specific variables and routine inclusion of pre and post-intervention measures of cognitive variables is important.

\section{Conclusions and next steps}

Once developed, an urgent question is: how best to deliver these techniques (dosage, mode) and to whom? Few studies have assessed individual differences in intervention responsiveness. Some assessed whether baseline symptoms moderated improvements but findings across studies were inconsistent [10, 19]. A handful of studies investigated individual differences in attention control [10] or purposeful engagement [64] but these findings require replication. Rather than personally tailoring interventions, an alternative approach is to develop an universal "toolkit" for young people; this could open up the possibility of having multiple techniques to choose from, which young people find appealing [85]. Consistent with this, and the combined cognitive bias hypothesis $[5,6]$, targeting several biases in interventions could yield benefits "greater than the sum of their parts". Combined approaches could be promising. Young people we consulted suggested that combining techniques to target 
attention and interpretation patterns could be helpful. Other strategies (e.g. recalling positive experiences) to amplify the use of helpful attention or interpretation patterns could also be beneficial. Delivering explicit instructions that encourage changes in strategic cognitive processes with reinforcement training to target more habitual ways of responding, could leverage interactive benefits across different levels of responding. For such multi-target, multi-level interventions to be sustainable, these will need co-design and co-evaluation with young people themselves. In addition, one lived experience representative suggested that a lack of recognition or understanding could affect usage of implementing these strategies. Therefore, it may also be useful to include psychoeducation or mental health literacy principles within these interventions, in order to help young people recognise the role of unhelpful thinking styles in everyday life and why there is a need to target these through helpful strategies.

\section{Acknowledgements}

We would like to thank Grace Williamson and Aleks Saunders for their help in double-coding some papers and their identification of lived experience consultants.

\section{Authors' contributions}

$J \mathrm{~L}, \mathrm{CH}$ and VP conceived of the specific questions explored in the systematic review. RW-M and IL conducted the search, selected studies and coded variables from each study. RW-M, IL and $\mathrm{JL}$ interpreted the findings and prepared the full paper draft. All authors read and approved the final manuscript.

\section{Funding}

This work was commissioned and carried out under the auspices of the Wellcome Trust's Mental Health Priority Area. The funding source had no role other than financial support.

\section{Availability of data and materials}

The datasets used and/or analysed during the current study are available from the corresponding author on reasonable request.

\section{Declarations}

\section{Consent for publications}

Not Applicable.

\section{Ethics approval and consent to participate}

As this was a systematic review, no primary data was collected. We sought young peoples' perspectives on the rationale of our research and on the nature of our findings but this was classed as Patient and Public Involvement and not research. Therefore we did not require ethical approval (Health Research Authority, 2020;https://www.hra.nhs.uk/planning-and-improvingresearch/best-practice/public-involvement/what-do-i-need-do/). However, all young people provided informed consent to be quoted with parental consent provided for young people under age 16 .

\section{Competing interests}

We have no financial or personal conflicts of interest to declare.

\section{Author details}

'Psychology Department, IOPPN, King's College London, Denmark Hill, London SE5 8AF, UK. ${ }^{2}$ Youth Resilience Research Unit, Queen Mary University of London, London, UK. ${ }^{3}$ Royal Holloway University of London, London, UK.
Received: 1 March 2021 Accepted: 19 May 2021

Published online: 25 August 2021

\section{References}

1. Lau JY, Waters AM. Annual research review: an expanded account of information-processing mechanisms in risk for child and adolescent anxiety and depression. J Child Psychol Psychiatry. 2017;58(4):387-407. https://doi. org/10.1111/jcpp.12653.

2. Beck AT, Haigh EA. Advances in cognitive theory and therapy: the generic cognitive model. Annu Rev Clin Psychol. 2014;10(1):1-24. https://doi.org/1 0.1146/annurev-clinpsy-032813-153734.

3. Beck AT, Dozois DJ. Cognitive therapy: current status and future directions Annu Rev Med. 2011;62(1):397-409. https://doi.org/10.1146/annurev-med052209-100032.

4. Haller SP, Cohen Kadosh K, Scerif G, Lau JY. Social anxiety disorder in adolescence: how developmental cognitive neuroscience findings may shape understanding and interventions for psychopathology. Dev Cogn Neurosci. 2015;13:11-20. https://doi.org/10.1016/j.dcn.2015.02.002.

5. Hirsch CR, Clark DM, Mathews A. Imagery and interpretations in social phobia: support for the combined cognitive biases hypothesis. Behav Ther. 2006;37(3):223-36. https://doi.org/10.1016/j.beth.2006.02.001.

6. Everaert J, Koster EH, Derakshan N. The combined cognitive bias hypothesis in depression. Clin Psychol Rev. 2012;32(5):413-24. https://doi.org/10.1016/j. cpr.2012.04.003.

7. Moher D, Liberati A, Tetzlaff J, Altman DG, Group P. Preferred reporting items for systematic reviews and meta-analyses: the PRISMA statement. Open Med. 2009;3(3):e123-30.

8. Yang $W$, Zhang JX, Ding Z, Xiao L. Attention Bias modification treatment for adolescents with major depression: a randomized controlled trial. J Am Acad Child Adolesc Psychiatry. 2016;55(3):208-18 e2. https://doi.org/10.101 6/j.jaac.2015.12.005.

9. Riemann BC, Kuckertz JM, Rozenman M, Weersing VR, Amir N. Augmentation of youth cognitive behavioral and pharmacological interventions with attention modification: a preliminary investigation. Depress Anxiety. 2013;30(9):822-8. https://doi.org/10.1002/da.22127.

10. Ollendick TH, White SW, Richey J, Kim-Spoon J, Ryan SM, Wieckowski AT, et al. Attention Bias modification treatment for adolescents with social anxiety disorder. Behav Ther. 2019;50(1):126-39. https://doi.org/10.1016/j. beth.2018.04.002.

11. Maoz K, Abend R, Fox NA, Pine DS, Bar-Haim Y. Subliminal attention bias modification training in socially anxious individuals. Front Hum Neurosci. 2013;7:389.

12. Yao N, Yu H, Qian M, Li S. Does attention redirection contribute to the effectiveness of attention bias modification on social anxiety? J Anxiety Disord. 2015;36:52-62. https://doi.org/10.1016/j.janxdis.2015.09.006.

13. Pan DN, Wang Y, Lei Z, Wang Y, Li X. The altered early components and the decisive later process underlying attention bias modification in social anxiety: evidence from event-related potentials. Soc Cogn Affect Neurosci. 2019;14(12):1307-16. https://doi.org/10.1093/scan/nsz098.

14. Liang CW, Hsu WY. Effects of attention bias modification with short and long stimulus-duration: a randomized experiment with individuals with subclinical social anxiety. Psychiatry Res. 2016;240:80-7. https://doi.org/10.1 016/j.psychres.2016.04.019.

15. Mastikhina L, Dobson K. Biased attention retraining in dysphoria: a failure to replicate. Cognit Emot. 2017;31(3):625-31. https://doi.org/10.1080/02 699931.2015 .1136270$.

16. Fitzgerald A, Rawdon C, Dooley B. A randomized controlled trial of attention bias modification training for socially anxious adolescents. Behav Res Ther. 2016;84:1-8. https://doi.org/10.1016/j.brat.2016.06.003.

17. De Voogd EL, Wiers RW, Salemink E. Online visual search attentional bias modification for adolescents with heightened anxiety and depressive symptoms: a randomized controlled trial. Behav Res Ther. 2017;92:57-67. https://doi.org/10.1016/j.brat.2017.02.006.

18. Bowler JO, Hoppitt L, Illingworth J, Dalgleish T, Ononaiye M, Perez-Olivas G, et al. Asymmetrical transfer effects of cognitive bias modification: modifying attention to threat influences interpretation of emotional ambiguity, but not vice versa. J Behav Ther Exp Psychiatry. 2017;54:239-46. https://doi.org/1 0.1016/j.jbtep.2016.08.011.

19. Baert S, De Raedt R, Schacht R, Koster EH. Attentional bias training in depression: therapeutic effects depend on depression severity. J Behav Ther Exp Psychiatry. 2010;41(3):265-74. https://doi.org/10.1016/j.jbtep.2010.02.004. 
20. Yang W, Ding Z, Dai T, Peng F, Zhang JX. Attention Bias Modification training in individuals with depressive symptoms: a randomized controlled trial. J Behav Ther Exp Psychiatry. 2015:49(Pt A):101-11.

21. Wells T, Beevers CG. Biased attention and dysphoria: manipulating selective attention reduces subsequent depressive symptoms. Cognit Emot. 2009; 24(4):719-28.

22. Dondzilo L, Rieger E, Shao R, Bell J. The effectiveness of touchscreen-based attentional bias modification to thin body stimuli on state rumination. Cognit Emot. 2020;34(5):1052-8. https://doi.org/10.1080/02699931.2020.171 8616.

23. Dai Q, Hu L, Feng Z. Attentional bias modification reduces clinical depression and enhances attention toward happiness. J Psychiatr Res. 2019; 109:145-55. https://doi.org/10.1016/j.jpsychires.2018.11.024.

24. Cai W, Pan Y, Chai H, Cui Y, Yan J, Dong W, et al. Attentional bias modification in reducing test anxiety vulnerability: a randomized controlled trial. BMC Psychiatry. 2018;18(1):1. https://doi.org/10.1186/s12888-017-1517-6

25. de Voogd EL, Wiers RW, Prins PJM, de Jong PJ, Boendermaker WJ, Zwitser $\mathrm{RJ}$, et al. Online attentional bias modification training targeting anxiety and depression in unselected adolescents: short- and long-term effects of a randomized controlled trial. Behav Res Ther. 2016;87:11-22. https://doi.org/1 0.1016/j.brat.2016.08.018

26. De Voogd EL, Wiers RW, Prins PJ, Salemink E. Visual search attentional bias modification reduced social phobia in adolescents. J Behav Ther Exp Psychiatry. 2014;45(2):252-9. https://doi.org/10.1016/j.jbtep.2013.11.006

27. Shomaker LB, Bruggink S, Pivarunas B, Skoranski A, Foss J, Chaffin E, et al. Pilot randomized controlled trial of a mindfulness-based group intervention in adolescent girls at risk for type 2 diabetes with depressive symptoms. Complement Ther Med. 2017;32:66-74. https://doi.org/10.1016/j.ctim.2017. 04.003.

28. Haukaas RB, Gjerde IB, Varting G, Hallan HE, Solem S. A randomized controlled trial comparing the attention training technique and mindful self-compassion for students with symptoms of depression and anxiety. Front Psychol. 2018;9:827. https://doi.org/10.3389/fpsyg.2018.00827.

29. Mogg K, Waters AM, Bradley BP. Attention Bias modification (ABM): review of effects of multisession ABM training on anxiety and threat-related attention in high-anxious individuals. Clin Psychol Sci. 2017;5(4):698-717. https://doi.org/10.1177/2167702617696359.

30. Yang R, Cui L, Li F, Xiao J, Zhang Q, Oei TPS. Effects of cognitive Bias modification training via smartphones. Front Psychol. 2017;8:1370. https:// doi.org/10.3389/fpsyg.2017.01370

31. Wells A. Metacognitive therapy for anxiety and depression. New York: The Guilford Press; 2009.

32. Bishop SR, Lau M, Shapiro S, Carlson L, Anderson ND, Jea C. Mindfulness: a proposed operational definition. Clin Psychol. 2004;11:230-41.

33. LeMoult J, Colich N, Joormann J, Singh MK, Eggleston C, Gotlib $1 \mathrm{H}$. Interpretation Bias training in depressed adolescents: near- and far-transfer effects. J Abnorm Child Psychol. 2018;46(1):159-67. https://doi.org/10.1007/ s10802-017-0285-6.

34. Micco JA, Henin A, Hirshfeld-Becker DR. Efficacy of interpretation Bias modification in depressed adolescents and young adults. Cognit Ther Res. 2014;38(2):89-102. https://doi.org/10.1007/s10608-013-9578-4.

35. Khalili-Torghabeh S, Fadardi JS, Mackintosh B, Reynolds S, Mobini S. Effects of a multi-session cognitive Bias modification program on interpretative biases and social anxiety symptoms in a sample of Iranian socially-anxious students. J Exp Psychopathol. 2014;5(4):514-27. https://doi.org/10.5127/jep. 037713

36. Butler E, Mobini S, Rapee RM, Mackintosh B, Reynolds SA. Enhanced effects of combined cognitive bias modification and computerised cognitive behaviour therapy on social anxiety. Cogent Psychol. 2015;2(1):1011905.

37. Klein AM, Salemink E, de Hullu E, Houtkamp E, Papa M, van der Molen M. Cognitive Bias modification reduces social anxiety symptoms in socially anxious adolescents with mild intellectual disabilities: a randomized controlled trial. J Autism Dev Disord. 2018;48(9):3116-26. https://doi.org/10.1 007/s10803-018-3579-9.

38. Smith HL, McDermott KA, Carlton CN, Cougle JR. Predictors of symptom outcome in interpretation Bias modification for dysphoria. Behav Ther. 2019; 50(3):646-58. https://doi.org/10.1016/j.beth.2018.10.001.

39. Albano AM, Marten PA, Holt CS, Heimberg RG, Barlow DH. Cognitivebehavioral group treatment for social phobia in adolescents: a preliminary study. J Nerv Ment Dis. 1995;183(10):649-56. https://doi.org/10.1097/ 00005053-199510000-00006.
40. Micco JA, Choate-Summers ML, Ehrenreich JT, Pincus DB, Mattis SG. Efficacious treatment components of panic control treatment for adolescents: a preliminary examination. Child Family Behav Ther. 2007;29(4): 1-23. https://doi.org/10.1300/J019v29n04_01.

41. Taheri E, Amiri M, Birashk B, Gharray B. Cognitive therapy versus behavioral activation therapy in the treatment of social anxiety disorder. J Fund Mental Health. 2016;18(5):294-9.

42. Topooco N, Berg M, Johansson S, Liljethorn L, Radvogin E, Vlaescu G, et al. Chat- and internet-based cognitive-behavioural therapy in treatment of adolescent depression: randomised controlled trial. BJPsych Open. 2018;4(4): 199-207. https://doi.org/10.1192/bjo.2018.18.

43. Ginsburg GS, Drake KL. School-based treatment for anxious african-american adolescents: a controlled pilot study. J Am Acad Child Adolesc Psychiatry. 2002;41(7):768-75. https://doi.org/10.1097/00004583-200207000-00007.

44. Rosenberg HJ, Jankowski MK, Fortuna LR, Rosenberg SD, Mueser KT. A pilot study of a cognitive restructuring program for treating posttraumatic disorders in adolescents. Psychol Trauma Theory Res Pract Policy. 2011;3(1): 94-9. https://doi.org/10.1037/a0019889.

45. Sportel BE, de Hullu E, de Jong PJ, Nauta MH. Cognitive bias modification versus CBT in reducing adolescent social anxiety: a randomized controlled trial. PLoS One. 2013;8(5):e64355. https://doi.org/10.1371/journal.pone.0064355.

46. Wessel I, Mersch PPA. A cognitive-behavioural group treatment for testanxious adolescents. Anxiety Stress Coping. 1994;7(2):149-60. https://doi. org/10.1080/10615809408249341

47. Hains AA, Davies WH, Parton E, Silverman AH. Brief report: a cognitive behavioral intervention for distressed adolescents with type I diabetes. Pediatr Psychol. 2001;26(1):61-6. https://doi.org/10.1093/jpepsy/26.1.61.

48. Deady M, Mills KL, Teesson M, Kay-Lambkin F. An online intervention for Cooccurring depression and problematic alcohol use in young people: primary outcomes from a randomized controlled trial. J Med Internet Res. 2016; 18(3):e71. https://doi.org/10.2196/jmir.5178.

49. Clarke GN, Hornbrook M, Lynch F, Polen M, Gale J, Beardslee W, et al. A randomized trial of a group cognitive intervention for preventing depression in adolescent offspring of depressed parents. Arch Gen Psychiatry. 2001;58(12):1127-34. https://doi.org/10.1001/archpsyc.58.12.1127.

50. Topooco N, Bylehn S, Dahlstrom Nysater E, Holmlund J, Lindegaard J, Johansson S, et al. Evaluating the efficacy of internet-delivered cognitive behavioral therapy blended with synchronous chat sessions to treat adolescent depression: randomized controlled trial. J Med Internet Res. 2019;21(11):e13393. https://doi.org/10.2196/13393.

51. Wright B, Tindall L, Littlewood E, Allgar V, Abeles P, Trepel D, et al. Computerised cognitive-behavioural therapy for depression in adolescents: feasibility results and 4-month outcomes of a UK randomised controlled trial. BMJ Open. 2017;7(1):e012834. https://doi.org/10.1136/bmjopen-2016-012834.

52. Fernandez NE, Mairal JB. Behavioral activation versus cognitive restructuring to reduce automatic negative thoughts in anxiety generating situations. Psicothema. 2017:29(2):172-7.

53. Sweeney GA, Horan JJ. Separate and combined effects of cue-controlled relaxation and cognitive restructuring in the treatment of musical performance anxiety. J Couns Psychol. 1982;29(5):486-97. https://doi.org/1 0.1037/0022-0167.29.5.486

54. Fu X, Du Y, Au S, Lau JYF. Single-session cognitive Bias modification of interpretations training in high-anxious adolescents. J Cogn Psychother. 2015;29(3):253-72. https://doi.org/10.1891/0889-8391.29.3.253.

55. Fu X, Du Y, Au S, Lau JY. Reducing negative interpretations in adolescents with anxiety disorders: a preliminary study investigating the effects of a single session of cognitive bias modification training. Dev Cogn Neurosci. 2013;4:29-37. https://doi.org/10.1016/j.den.2012.11.003.

56. Fremouw WJ, Zitter RE. A comparison of skills training and cognitive restructuring-relaxation for the treatment of speech anxiety. Behav Ther. 1978;9(2):248-59. https://doi.org/10.1016/S0005-7894(78)80110-5.

57. Gross RT, Fremouw WJ. Cognitive restructuring and progressive relaxation for treatment of empirical subtypes of speech-anxious subjects. Cogn Ther Res. 1982;6(4):429-36. https://doi.org/10.1007/BF01184009.

58. Decker TW, Russell RK. Comparison of cue-controlled relaxation and cognitive restructuring versus study skills counseling in treatment of testanxious college underachievers. Psychol Rep. 1981;49(2):459-69. https://doi. org/10.2466/pro.1981.49.2.459.

59. Bistline $J$, Jaremko ME, Sobleman $\mathrm{S}$. The relative contributions of covert reinforcement and cognitive restructuring to test anxiety reduction. J Clin Psychol. 1980;36(3):723-8. 
60. Wise EH, Haynes SN. Cognitive treatment of test anxiety: rational restructuring versus attentional training. Cogn Ther Res. 1983;7(1):69-77. https://doi.org/10.1007/BF01173425.

61. Arnkoff DB. A comparison of the coping and restructuring components of cognitive restructuring. Cogn Ther Res. 1986;10(2):147-58. https://doi.org/1 0.1007/BF01173721.

62. Clore J, Scott S. Self-statement modification techniques for distressed college students with low self-esteem and depressive symptoms. Int $J$ Behav Consult Ther. 2006;2(3):314-31. https://doi.org/10.1037/h0100786.

63. Stevens ES, Behar E, Jendrusina AA. Enhancing the efficacy of cognitive Bias modification for social anxiety. Behav Ther. 2018;49(6):995-1007. https://doi. org/10.1016/j.beth.2018.02.004.

64. Rodebaugh $\mathrm{TL}$, Jakatdar TA, Rosenberg A, Heimberg RG. Thinking about social situations: the moderated effects of imposing structure. Behav Res Ther. 2009;47(2):158-63. https://doi.org/10.1016/j.brat.2008.10.019.

65. de Voogd L, Wiers RW, de Jong PJ, Zwitser RJ, Salemink E. A randomized controlled trial of multi-session online interpretation bias modification training: short- and long-term effects on anxiety and depression in unselected adolescents. PLoS One. 2018;13(3):e0194274. https://doi.org/1 0.1371/journal.pone.0194274.

66. Chan SW, Lau JY, Reynolds SA. Is cognitive bias modification training truly beneficial for adolescents? J Child Psychol Psychiatry. 2015;56(11):1239-48. https://doi.org/10.1111/jcpp.12368.

67. Lothmann C, Holmes EA, Chan SW, Lau JY. Cognitive bias modification training in adolescents: effects on interpretation biases and mood. J Child Psychol Psychiatry. 2011;52(1):24-32. https://doi.org/10.1111/j.1469-7610.201 0.02286.x.

68. Belli S, Lau JY. Cognitive Bias modification training in adolescents: persistence of training effects. Cogn Ther Res. 2014;38(6):640-51. https://doi. org/10.1007/s10608-014-9627-7

69. Hoppitt L, Illingworth JL, MacLeod C, Hampshire A, Dunn BD, Mackintosh B. Modifying social anxiety related to a real-life stressor using online cognitive Bias modification for interpretation. Behav Res Ther. 2014;52:45-52. https:// doi.org/10.1016/j.brat.2013.10.008

70. Telman MD, Holmes EA, Lau JY. Modifying adolescent interpretation biases through cognitive training: effects on negative affect and stress appraisals. Child Psychiatry Hum Dev. 2013;44(5):602-11. https://doi.org/10.1007/s1 0578-013-0386-6.

71. Lau JY, Belli SR, Chopra RB. Cognitive bias modification training in adolescents reduces anxiety to a psychological challenge. Clin Child Psychol Psychiatry. 2013;18(3):322-33. https://doi.org/10.1177/1359104512455183.

72. Salemink E, Wiers RW. Modifying threat-related interpretive bias in adolescents. J Abnorm Child Psychol. 2011;39(7):967-76. https://doi.org/10.1 007/s10802-011-9523-5.

73. Lau JY, Molyneaux E, Telman MD, Belli S. The plasticity of adolescent cognitions: data from a novel cognitive bias modification training task. Child Psychiatry Hum Dev. 2011;42(6):679-93. https://doi.org/10.1007/s10578-0110244-3.

74. Rodriguez LM, Lee KDM, Onufrak J, Dell JB, Quist M, Drake HP, et al. Effects of a brief interpersonal conflict cognitive reappraisal intervention on improvements in access to emotion regulation strategies and depressive symptoms in college students. Psychol Health. 2020;1:1-21.

75. Kiselica MS, Baker SB, Thomas RN, Reedy S. Effects of stress inoculation training on anxiety, stress, and academic performance among adolescents. J Couns Psychol. 1994;41(3):335-42. https://doi.org/10.1037/0022-0167.41.3.335.

76. Hains AA. A stress inoculation training program for adolescents in a high school setting: a multiple baseline approach. J Adolesc. 1992;15(2):163-75. https://doi.org/10.1016/0140-1971(92)90045-7.

77. Hains AA, Ellmann SW. Stress inoculation training as a preventative intervention for high school youths. J Cogn Psychother. 1994;8(3):219-32. https://doi.org/10.1891/0889-8391.8.3.219.

78. Rohsenow DJ, Smith RE, Johnson S. Stress management training as a prevention program for heavy social drinkers: cognitions, affect, drinking, and individual differences. Addict Behav. 1985;10(1):45-54. https://doi.org/1 0.1016/0306-4603(85)90052-8.

79. Hains AA, Szyjakowski M. A cognitive stress-reduction intervention program for adolescents. J Couns Psychol. 1990;37(1):79-84. https://doi.org/10.1037/ 0022-0167.37.1.79.

80. Lehenbauer M, Kothgassner OD, Kryspin-Exnera I, Stetinab BU. An online self-administered social skills training for young adults: results from a pilot study. Comput Educ. 2013;61:217-24. https://doi.org/10.1016/j.compedu.2 012.09.007.

81. Waters AJ, Donaldson J, Zimmer-Gembeck MJ. Cognitive-behavioural therapy combined with an interpersonal skills component in the treatment of generalised anxiety disorder in adolescent females: a case series. Behav Chang. 2008;25(1):35-43. https://doi.org/10.1375/bech.25.1.35.

82. McCall HC, Richardson CG, Helgadottir FD, Chen FS. Evaluating a web-based social anxiety intervention Among University students: randomized controlled trial. J Med Internet Res. 2018;20(3):e91. https://doi.org/10.2196/ jmir.8630.

83. Piet J, Hougaard E, Hecksher MS, Rosenberg NK. A randomized pilot study of mindfulness-based cognitive therapy and group cognitive-behavioral therapy for young adults with social phobia. Scand J Psychol. 2010;51(5): 403-10. https://doi.org/10.1111/j.1467-9450.2009.00801.x.

84. O'Toole MS, Renna ME, Mennin DS, Fresco DM. Changes in decentering and reappraisal temporally precede symptom reduction during emotion regulation therapy for generalized anxiety disorder with and without cooccurring depression. Behav Ther. 2019;50(6):1042-52. https://doi.org/10.101 6/j.beth.2018.12.005.

85. Loucas CE, Sclare I, Stahl D, Michelson D. Feasibility randomized controlled trial of a one-day CBT workshop ('DISCOVER') for 15- to 18-year-olds with anxiety and/or depression in clinic settings. Behav Cogn Psychother. 2020; 48(2):142-59. https://doi.org/10.1017/S1352465819000286.

86. Lisk SC, Pile V, Haller SPW, Kumari V, Lau JYF. Multisession cognitive Bias modification targeting multiple biases in adolescents with elevated social anxiety. Cognit Ther Res. 2018;42(5):581-97. https://doi.org/10.1007/s10608018-9912-y.

87. Lau JYF, Sharma NP, Bennett E, Dhakal S, Vaswani A, Pandey R, et al. Acceptability of a brief training programme targeting attention and interpretation biases for threat in youth with a history of maltreatment. Behav Cogn Psychother. 2020;48(3):370-5. https://doi.org/10.1017/S13524 65819000663.

88. Stuijfzand S, Creswell C, Field AP, Pearcey S, Dodd H. Research review: is anxiety associated with negative interpretations of ambiguity in children and adolescents? A systematic review and meta-analysis. J Child Psychol Psychiatry. 2018;59(11):1127-42. https://doi.org/10.1111/jcpp.12822.

89. Dudeney J, Sharpe L, Hunt C. Attentional bias towards threatening stimuli in children with anxiety: a meta-analysis. Clin Psychol Rev. 2015;40:66-75. https://doi.org/10.1016/j.cpr.2015.05.007.

90. Waters AM, Pittaway M, Mogg K, Bradley BP, Pine DS. Attention training towards positive stimuli in clinically anxious children. Dev Cogn Neurosci. 2013;4:77-84. https://doi.org/10.1016/j.dcn.2012.09.004.

91. Waters AM, Zimmer-Gembeck MJ, Craske MG, Pine DS, Bradley BP, Mogg K. Look for good and never give up: a novel attention training treatment for childhood anxiety disorders. Behav Res Ther. 2015;73:111-23. https://doi. org/10.1016/j.brat.2015.08.005.

92. Lazarov A, Pine DS, Bar-Haim Y. Gaze-contingent music reward therapy for social anxiety disorder: a randomized controlled trial. Am J Psychiatry. 2017; 174(7):649-56. https://doi.org/10.1176/appi.ajp.2016.16080894.

93. Shamai-Leshem D, Lazarov A, Pine DS, Bar-Haim Y. A randomized controlled trial of gaze-contingent music reward therapy for major depressive disorder. Depress Anxiety. 2020;38(2):134.

94. Linetzky M, Kahn M, Lazarov A, Pine DS, Bar-Haim Y. Gaze-contingent music reward therapy for clinically anxious 7- to 10-year-olds: an open multiple baseline feasibility study. J Clin Child Adolesc Psychol. 2019;1:1-8.

95. Hirsch CR, Krahe C, Whyte J, Bridge L, Loizou S, Norton S, et al. Effects of modifying interpretation bias on transdiagnostic repetitive negative thinking. J Consult Clin Psychol. 2020;88(3):226-39. https://doi.org/10.1037/ ccp0000455.

96. Valmaggia LR, Freeman D, Green C, Garety P, Swapp D, Antley A, et al. Virtual reality and paranoid ideations in people with an 'at-risk mental state' for psychosis. Br J Psychiatry Suppl. 2007;51:s63-8. https://doi.org/10.1192/ bjp.191.51.s63.

\section{Publisher's Note}

Springer Nature remains neutral with regard to jurisdictional claims in published maps and institutional affiliations. 